\title{
Correlation between impedance cardiography and 6 min walk distance in atrial fibrillation patients
}

Ling Ding ${ }^{\dagger}$, Xiao-Qing Quan ${ }^{\dagger}$, Shu Zhang ${ }^{\dagger}$, Lei Ruan, Le Zhang, Kai Zheng, Wei-Wei Yu, Xiao-Fen Wu, Tao Mi, Cun-Tai Zhang and Hong-Lian Zhou*

\begin{abstract}
Background: The correlation between impedance cardiography (ICG) and 6 min walk distance (6MWD) in atrial fibrillation (AF) patients remains unknown.

Methods: We recruited 49 subjects in the study (21 AF patients and 28 patients without AF) and estimated hemodynamic parameters: cardiac output (CO), stroke volume (SV), stroke volume index (SVI), left stroke work (LSW), left stroke work index (LSWI), stroke systemic vascular resistance (SSVR), stroke systemic vascular resistance index (SSVRI); 6MWD, left ventricle ejection fraction (LVEF), NT-pro brain natriuretic peptide (NT-pro BNP) for the two groups.

Results: The AF group have apparently lower CO $(2.26 \pm 0.14 \mathrm{VS} 4.11 \pm 0.20 \mathrm{~L} / \mathrm{min}, p=0.039)$ and distinctly higher SVR (677.60 \pm 69.10 VS $344.41 \pm 22.98$ dynes/cm $\left.\mathrm{cm}^{5}, p=0.001\right)$, SSVRI $\left(396.97 \pm 36.80\right.$ VS $199.01 \pm 11.72$ dynes $/ \mathrm{cm}^{5} / \mathrm{m}^{2}, p<0$. 001) than the control group. NT-pro BNP $(1409.48 \pm 239.90 \mathrm{VS} 332.59 \pm 68.85 \mathrm{pg} / \mathrm{ml}, p=0.001)$ in the AF group was significantly higher than the control group and 6MWD (264.33 \pm 14.55 VS $428.79 \pm 29.98 \mathrm{~m}, p<0.001)$ in the AF group was lower than the control group. There was no significant difference in LVEF between the two groups $(62.67 \pm 7.62 \%$ VS $63.93 \pm 5.03 \%, p=0.470)$. Pearson correlation analysis revealed that $C O(R=0.494, p=0.023)$, SV $(R=0.633, p=0.002), \mathrm{LSW}(R=0.615, p=0.003)$ and $\mathrm{LSWI}(R=0.491, p=0.024)$ significantly correlated positively with $6 \mathrm{MWD}$ in AF patients.

Conclusions: AF patients had lower cardiac output, shorter 6MWD and higher NT-pro BNP than patients with sinus rhythm. The cardiac output measured by impedance cardiography significantly correlated positively with 6MWD in AF patients.
\end{abstract}

Keywords: Impedance cardiography, 6 min walk distance, Atrial fibrillation

\section{Backgrounds}

Atrial fibrillation (AF) is a kind of common cardiac arrhythmia [1], which disrupts the movement of the left atrial. AF is defined as an irregular, disorganized electrical activity of the atria and it is a growing clinical problem associated with increased morbidity and mortality $[2,3]$. Estimating cardiac function in AF patients contributes to judge prognosis and adjust therapeutic schedule. Traditionally, left ventricle ejection fraction

\footnotetext{
*Correspondence: zhouhonglian63@163.com

${ }^{\dagger}$ Equal contributors

Department of Geriatrics, Tongji Hospital, Tongji Medical College, Huazhong University of Science and Technology, Wuhan 430030, China
}

(LVEF), the fraction of outbound blood pumped from the heart with each heartbeat measured by cardiac color ultrasound (CCU) has been chosen to assess the cardiac function in patients including AF. However, stroke volume changed in different cardiac cycles because of irregular diastole and systole of the atria [3, 4]. Lacking of an active contraction of the LA (atrial kick) in late diastole, cardiac output in AF patients may be less than in normal persons. LVEF measured by CCU only reflect cardiac function of contraction in one cardiac cycle, not the whole cardiac function in AF patients.

A considerable proportion of previous data have stated the role of hemodynamic parameters measured by impedance cardiography (ICG) in diagnostic, prognostic, 
and therapeutic decision making in chronic heart failure [5-7] and pulmonary hypertension [8]. ICG is a reliable, noninvasive technique that can be easily used to continually measure hemodynamic parameters and output the mean values [9]. ICG is based on the Ohm's law stating that when a constant current travels through a conductor, changes in voltage are directly proportional to variations in impedance [9]. Bio-impedance decreases across the chest during systole as a result of an increase in aortic blood volume and flow velocity. There are series of hemodynamic parameters associated with cardiac function such as cardiac output (CO), stroke volume (SV), stroke volume index (SVI), left stroke work (LSW), left stroke work index (LSWI), stroke systemic vascular resistance (SSVR) and stroke systemic vascular resistance index (SSVRI) measured by ICG.

Gilewski et al. investigated cardiovascular changes in tissue Doppler echocardiography (TDE) and ICG in AF patients subjected to cardioversion [10]. The study suggest that ICG seems to be complementary to standard echocardiography, and to some extent to surpass TDE. However, there is little published study on the correlation between ICG and 6 min walk distance (6MWD) or the value of ICG in predicting the cardiac function in AF patients. Although AF patients have irregular diastole period, ICG could continually measure parameters related to cardiac function, which provides more accurate information to predict the cardiac condition in AF patients. The present study will research the values of impedance cardiography in predicting cardiac function and the correlation between hemodynamic parameters and 6MWD in AF patients.

\section{Methods}

\section{Study sample}

This study was approved by the Clinical Research Ethics Committee of Tongji Hospital, Tongji Medical College, Huazhong University of Science and Technology. All participating persons were given informed consent and written informed consent was obtained from all subjects before enrollment. The study was performed between January 2014 and December 2015. We recruited patients with $\operatorname{AF}(n=21)$ and healthy controls $(n=28)$. All the AF patients in our study were persistent or longstanding persistent AF according to 2014 AHA/ACC/HRS guideline [11]. Patients in healthy control group were sinus rhythm confirmed by electrocardiogram and ambulatory electrocardiogram. Patients in the two groups were matching for age, gender, height, weight, body mass index (BMI), underlying disease and medicine.

Exclusion criteria were impaired cognition, chronic obstructive pulmonary diseases (COPD), hyperthyroidism, severe hepatic disease, severe renal impairment, asthma, cystic, fibrosis, arthritis, ankle, knee or hip injuries, muscle wasting, any life-threatening disease, drugs and/or alcohol abuse $[12,13]$. Additionally, we excluded patients with pacemaker implantation, valvular heart disease, enlarged LV dimension, recent myocardial infarction, unstable angina, candidacy for revascularization, cardiomyopathy, congestive heart failure, pulmonary heart disease, a resting heart rate of more than $120 \mathrm{bpm}$, a systolic blood pressure of more than $180 \mathrm{mmHg}$, and a diastolic blood pressure of more than $100 \mathrm{mmHg}[10,12,13]$.

\section{Data collection}

ICG was performed using the Cheer Sails Medical (CSM3000 system) with electrodes placed on the neck and in the hypochondriac regions. The basic principle of this methodology is that variations in the impedance $(\mathrm{Z})$ to an alternating high-frequency $(75 \mathrm{KHz})$ and low magnitude $(1.8 \mathrm{~mA})$ current, across the thorax during cardiac ejection, result in a specific waveform that can be used to calculate stroke volume [14]. After $5 \mathrm{~min}$ of rest in the supine position in an examination room, a technician performed ICG. The hemodynamic variables $\mathrm{CO}$, SV, SVI, LSW, LSWI, SVR, and SVRI referring to the cardiac function and health condition were showed. Values of the hemodynamic parameters represented the mean value over the 3-minute sampling period.

CCU was performed with the aim of measuring LVEF. Diastolic left ventricular diameter (DLVD), left ventricular posterior wall thickness (LVPWT), interventricular septal thickness (IVST) and left atrial diameter (LAD) were also recorded. The 6MWT was performed according to standard guideline [12]. A single walk test without practice was administered. The 6 min walk test (6MWT) was ordered by the patient's physicians as an initial or follow-up assessment of their cardiac function according to Automatic Test System (ATS) standards [12, 13]. All distance data were recorded accurately. NT-pro BNP were tested by picking venous blood in clinical laboratory.

\section{Data analysis}

Baseline subject characteristics across quartiles were compared using independent-samples $t$ test for continuous variables and Pearson $\chi^{2}$ test for dichotomous variables $[15,16]$. All the statistic values of continuous variables was mean \pm standard error. In $\chi^{2}$ test, continuity correction was for dichotomous variables with fewer than 5 participants in a categories. Statistical significance was set to a $\mathrm{p}$ value less than 0.05 . The strength of association between variables was performed using Pearson correlation test [17].

\section{Results}

Baseline characteristics of the AF group and the control group were presented in Table 1 . The subjects in the two groups had no statistical difference in age, gender, 
Table 1 Baseline characteristics of all the subjects

\begin{tabular}{|c|c|c|c|c|}
\hline Parameters & Control group $(n=28)$ & AF group $(n=21)$ & $t / x^{2}$ & $P$ \\
\hline Age (years) & $75.36 \pm 2.23$ & $83.19 \pm 2.0$ & 2.523 & 0.093 \\
\hline Gender, n (\% Male) & $20(71.4)$ & $17(81.0)$ & 0.589 & 0.443 \\
\hline Height (cm) & $166.07 \pm 1.79$ & $164.76 \pm 1.47$ & -0.540 & 0.043 \\
\hline Weight (kg) & $65.25 \pm 2.184$ & $61.57 \pm 2.260$ & -1.152 & 0.421 \\
\hline $\mathrm{BMI}\left(\mathrm{kg}^{2} / \mathrm{m}\right)$ & $23.52 \pm 0.52$ & $22.68 \pm 0.79$ & -0.929 & 0.191 \\
\hline Heart rate (bpm) & $68.50 \pm 1.99$ & $68.90 \pm 2.24$ & 0.135 & 0.890 \\
\hline SBP $(\mathrm{mmHg})$ & $120.57 \pm 2.91$ & $120.43 \pm 3.83$ & -0.030 & 0.676 \\
\hline $\mathrm{DBP}(\mathrm{mmHg})$ & $67.25 \pm 1.73$ & $69.00 \pm 1.63$ & 0.715 & 0.279 \\
\hline Hypertension, n (\%) & $20(71.4)$ & $18(85.7)$ & 0.706 & 0.401 \\
\hline $\mathrm{T}_{2} \mathrm{DM}, \mathrm{n}(\%)$ & $4(14.3)$ & $3(14.3)$ & 0 & 1.000 \\
\hline CRD, $n(\%)$ & $2(7.1)$ & $1(4.8)$ & 0.118 & 1.000 \\
\hline$C A D, n(\%)$ & $4(14.3)$ & $2(9.5)$ & 0.253 & 0.688 \\
\hline$\beta$-blocker treatment, $\mathrm{n}(\%)$ & $8(28.6)$ & $7(33.3)$ & 0.128 & 0.720 \\
\hline Digoxin treatment, $\mathrm{n}(\%)$ & $0(0)$ & $5(23.8)$ & 5.053 & 0.025 \\
\hline
\end{tabular}

Abbreviations: $B M I$ body mass index, SBP systolic blood pressure, $D B P$ diastolic blood pressure, T2DM type 2 diabetes mellitus, CRD chronic renal disease, $C A D$ coronary artery disease

weight, BMI, or underlying disease such as diabetes mellitus and hypertension or basic medicine such as $\beta$-receptor blocker. There were five $(23.8 \%)$ patients in the AF group using digoxin and nobody in the control group using the same medicine, which made statistical difference in the two groups.

Table 2 defined the comparison of hemodynamic parameters, 6MWD, LVEF and NT-pro BNP between the AF group and the control group. The present study found that the 6MWD in AF patients was shorter than the control group $(264.33 \pm 14.55$ VS $428.79 \pm 29.98 \mathrm{~m}$, $p<0.001)$. The patients in the AF group had apparently lower CO $(2.26 \pm 0.14 \mathrm{VS} 4.11 \pm 0.20 \mathrm{~L} / \mathrm{min}, p=0.039)$ and distinctly higher SVR $(677.60 \pm 69.10 \mathrm{VS} 344.41 \pm$ 22.98 dynes $\left./ \mathrm{cm}^{5}, p=0.001\right)$, SSVRI $(396.97 \pm 36.80 \mathrm{VS}$ $199.01 \pm 11.72$ dynes $\left./ \mathrm{cm}^{5} / \mathrm{m}^{2}, p<0.001\right)$ than those in the control group. NT-pro BNP (1409.48 $\pm 239.90 \mathrm{VS}$ $332.59 \pm 68.85 \mathrm{pg} / \mathrm{ml}, p=0.001)$ in the AF group was significantly higher than the control group. There was no significant difference in LVEF between the two groups $(62.67 \pm 7.62 \%$ VS $63.93 \pm 5.03 \%, p=0.470)$. No significant difference was observed in other measured echocardiographic parameters (Table 2).

As shown in Table 3, Pearson correlation analysis revealed that $\mathrm{CO}(\mathrm{R}=0.494, p=0.023), \mathrm{SV}(\mathrm{R}=0.633$, $p=0.002)$, LSW $(\mathrm{R}=0.615, p=0.003)$ and LSWI $(\mathrm{R}=$ $0.491, p=0.024$ ) significantly correlated positively with 6MWD. LVEF did not significantly correlate with 6MWD $(\mathrm{R}=0.037, p=0.803)$.

\section{Discussion}

In previous published study, non-invasive impedance cardiography has been used to reveal heterogeneity of hemodynamic parameters in primary hypertension $[18,19]$ and guide antihypertensive therapy [19-21]. However, there is no published data studying the role of impedance cardiography in patients with atrial fibrillation or the correlation between hemodynamic parameters and 6MWD. In the present study, we explored the feature of hemodynamic parameters in AF patients and statistical correlation among hemodynamic parameters, LVEF, NT-pro BNP and 6MWD.

$6 \mathrm{MWT}$ is used to measure the distance that a patient can quickly walk on a flat, hard surface in a period of $6 \mathrm{~min}$ [12], and 6MWD is the distance in 6MWT. Due to wide availability, security and ease of implementation, $6 \mathrm{MWT}$ had been used in the assessment of functional capacity, evaluating exercise tolerance, prognosis and therapeutic effectiveness in patients with impaired cardiac function [22-27]. The present study found that 6MWD in AF patients was shorter than the control group $(264.33 \pm 14.55$ VS $428.79 \pm 29.98 \mathrm{~m}, p<0.001)$ whereas LVEF had no significant difference between the AF group and the control group $(62.67 \pm 7.62 \%$ VS $63.93 \pm 5.03 \%, p=0.470)$. Our study also found that LVEF did not correlate with 6MWD $(\mathrm{R}=0.037, p=0.803)$. The result suggested that LVEF could not predict cardiac function accurately in AF patients.

BNP levels could be higher in patients with impaired cardiac function $[26,28]$. The present data showed that AF patients had significantly higher NT-pro BNP than the control group (1385.74 \pm 1102.51 VS 404.54 \pm $322.33 \mathrm{pg} / \mathrm{ml}, p<0.001)$. Although the value of LVEF in AF patients remained normal in the present study, the cardiac function in the AF group was worse than the control group according to the 6MWD and NT-pro BNP. 
Table 2 Comparison of hemodynamic, 6MWD, LVEF, NT-pro BNP between the control group and the AF group

\begin{tabular}{|c|c|c|c|c|}
\hline Parameter & Control group $(n=28)$ & AF group $(n=21)$ & $\mathrm{t}$ & $p$ \\
\hline \multicolumn{5}{|l|}{ ICG parameters } \\
\hline $\mathrm{CO}(\mathrm{L} / \mathrm{min})$ & $4.11 \pm 0.20$ & $2.26 \pm 0.14$ & 4.502 & 0.039 \\
\hline $\mathrm{SV}(\mathrm{mL})$ & $60.28 \pm 3.30$ & $32.45 \pm 2.55$ & 0.885 & 0.352 \\
\hline $\mathrm{SVI}\left(\mathrm{mL} / \mathrm{m}^{2}\right)$ & $34.93 \pm 1.61$ & $18.89 \pm 1.74$ & 0.086 & 0.771 \\
\hline SVR (dynes/cm5) & $344.41 \pm 22.98$ & $677.60 \pm 69.10$ & 11.549 & 0.001 \\
\hline SVRI (dynes $/ \mathrm{cm}^{5} / \mathrm{m}^{2}$ ) & $199.01 \pm 11.72$ & $396.97 \pm 36.80$ & 14.556 & $<0.001$ \\
\hline LSW (gm-m/beat) & $66.42 \pm 3.72$ & $37.03 \pm 2.77$ & 3.429 & 0.070 \\
\hline LSWI (gm-m/m²/beat) & $38.96 \pm 1.85$ & $22.42 \pm 1.59$ & 2.421 & 0.126 \\
\hline \multicolumn{5}{|l|}{ CCU parameters } \\
\hline LVEF (\%) & $64.57 \pm 0.92$ & $63.05 \pm 1.84$ & 3.307 & 0.075 \\
\hline $\mathrm{LAD}(\mathrm{mm})$ & $33.86 \pm 1.00$ & $40.67 \pm 1.71$ & 3.634 & 0.164 \\
\hline DLVD (mm) & $44.96 \pm 0.72$ & $46.67 \pm 1.44$ & 1.140 & 0.056 \\
\hline IVST (mm) & $10.21 \pm 0.24$ & $9.95 \pm 0.24$ & 0.781 & 0.586 \\
\hline LVPWT (mm) & $9.89 \pm 0.22$ & $9.75 \pm 0.20$ & 0.489 & 0.981 \\
\hline \multicolumn{5}{|l|}{ Other parameters } \\
\hline NT-pro BNP (pg/ml) & $332.59 \pm 68.85$ & $1409.48 \pm 239.90$ & 13.470 & 0.001 \\
\hline 6MWD (m) & $428.79 \pm 29.98$ & $264.33 \pm 14.55$ & 16.816 & $<0.001$ \\
\hline
\end{tabular}

Abbreviations: AF atrial fibrillation, ICG impedance cardiography, 6MWD 6 min walk distance, LVEF left ventricle ejection fraction, NT-pro BNP plasma NT-pro brain natriuretic peptide, CO cardiac output, SV stroke volume, SVI stroke volume index, SSVR stroke systemic vascular resistance, SSVRI stroke systemic vascular resistance index, LSW left stroke work, LSWI left stroke work index, CCU cardiac color ultrasound, LAD left atrial diameter, DLVD diastolic left ventricular diameter, IVST interventricular septal thickness, LVPWT left ventricular posterior wall thickness

Due to great reliability and validity, ICG had been used in the diagnosis, prognosis and therapy in a variety of diseases [20, 21]. A recent study of AF patients subjected to cardioversion suggests that ICG seems to be complementary to standard echocardiography, and to some extent to surpass TDE [10]. The present study discovered that the CO $(2.26 \pm 0.14 \mathrm{VS} 4.11 \pm 0.20 \mathrm{~L} / \mathrm{min}, p=0.039)$ measured by impedance cardiography in AF patients were lower than the control group. Our data also found that CO ( $\mathrm{R}=0.494, p=0.023)$, SV $(\mathrm{R}=0.633, p=0.002), \mathrm{LSW}$ $(\mathrm{R}=0.615, p=0.003)$ and LSWI $(\mathrm{R}=0.491, p=0.024)$ correlated positively with 6MWD. Lacking of the atrial

Table 3 Correlation between hemodynamic parameters, LVEF and 6MWD in all patients

\begin{tabular}{lclcl}
\hline Parameters & Control group R & $p$ & AF group R & $P$ \\
\hline CO $(\mathrm{L} / \mathrm{min})$ & 0.626 & $<0.001$ & 0.494 & 0.023 \\
SV $(\mathrm{mL})$ & 0.587 & 0.001 & 0.633 & 0.002 \\
SVI $\left(\mathrm{mL} / \mathrm{m}^{2}\right)$ & 0.518 & 0.005 & 0.512 & 0.018 \\
SVR (dynes $\left./ \mathrm{cm}^{5}\right)$ & -0.261 & 0.179 & -0.331 & 0.143 \\
SVRI (dynes $\left./ \mathrm{cm}^{5} / \mathrm{m}^{2}\right)$ & -0.389 & 0.041 & -0.413 & 0.063 \\
LSW (gm-m/beat) & 0.488 & 0.008 & 0.615 & 0.003 \\
LSWI (gm-m/m $/ \mathrm{m} /$ beat) & 0.488 & 0.008 & 0.491 & 0.024 \\
LVEF (\%) & 0.106 & 0.591 & -0.307 & 0.176 \\
NT-pro BNP (pg/ml) & -0.503 & 0.006 & 0.378 & 0.091 \\
\hline
\end{tabular}

Abbreviation: $R$ Correlation coefficient kick in late diastole [4], the cardiac output in AF patients was lower than the non AF patients. With lower cardiac output, the AF patients generally have shorter 6MWD.

6MWD had been proved to be useful and reliable in the assessment of functional capacity, evaluating exercise tolerance, prognosis and therapeutic effectiveness in patients with impair cardiac function [22, 29, 30]. The present study detected that hemodynamic parameters had a significant correlation with the 6MWD whereas LVEF measurements correlated poorly with the 6MWD in AF patients. The results of this study suggest that hemodynamic parameters measured by ICG could provide more accurate information than LVEF to predict cardiac function in AF patients. It is noteworthy that all the $\mathrm{AF}$ patients in our study were persistent or longstanding persistent AF, and we excluded AF patients with obviously abnormal heart structure. This strengthen the novelty of the present study.

\section{Clinical implications}

Digitalis and beta-blockers have long been used to control heart rate in AF patients in order to reduce AF-related symptoms [31, 32]. The main hemodynamic change following the treatment with beta-blockers is a decrease of cardiac output. Digoxin therapy could increase the cardiac output and offer symptomatic improvement in AF patients with decreased cardiac output and cardiac function $[33,34]$. Therefore, evaluating the cardiac output and 
cardiac function in AF patients is important. Although the value of LVEF in AF patients remained normal in the present study, the cardiac function in the AF patients was worse than the control group according to the 6MWD and NT-pro BNP. Our study also found that cardiac output measured by ICG significantly correlated positively with 6MWD in AF patients, and LVEF did not correlate with 6MWD. Clinical doctors may adjust therapeutic schedule of digitalis and beta-blockers according to the information from ICG. This is just our speculation according to our finding and the specific application need further investigation.

\section{Study limitations}

Our study has limitations: (1) The study sample was relatively small, limiting the statistical power of group analyses. (2) The subjects in our study were relatively old and their parameters maybe could not represent the condition of the whole population. (3) We did not provide some important echocardiographic parameters such as cardiac index and left ventricular end-diastolic volume. Whether the diastolic dysfunction contribute to the decreased cardiac output in AF patients is not clear. Further studies are needed to determine the mechanism underlying the hemodynamic disorders observed in AF.

\section{Conclusion}

Compared to patients with sinus rhythm, AF patients had lower cardiac output, shorter 6MWD and higher NT-pro BNP. The cardiac output measured by impedance cardiography significantly correlated positively with 6MWD in AF patients.

\footnotetext{
Abbreviations

$6 \mathrm{MWD}, 6$ min walk distance; $6 \mathrm{MWT}, 6$ min walk test; AF, atrial fibrillation; ATS, Automatic Test System; BMI, body mass index; CCU, cardiac color ultrasound; CO, cardiac output; COPD, chronic obstructive pulmonary diseases; DLVD, diastolic left ventricular diameter; EDV, end-diastolic volume; ICG, impedance cardiography; IVST, interventricular septal thickness; LAD, left atrial diameter; LSW, left stroke work; LSWI, left stroke work index; LVEF, left ventricle ejection fraction; LVPWT, left ventricular posterior wall thickness; NT-pro BNP, NT-pro brain natriuretic peptide; SSVR, stroke systemic vascular resistance; SSVRI, stroke systemic vascular resistance index; SV, stroke volume; SVI, stroke volume index
}

\section{Acknowledgements \\ The authors thank the grants from the National Natural Science Foundation of China and the Scientific and Technological Projects of Hubei. The funders had no role in the study design, data collection, analysis, decision to publish, or preparation of the manuscript. The corresponding author had full access to all the data in the study and had final responsibility for the decision to submit for publication.}

\section{Funding}

This work was supported by the grants from the National Natural Science Foundation of China $(81400255,81300141)$, and the Scientific and Technological Projects of Hubei (2015CFB624).

\section{Availability of data and materials}

All relevant data supporting the conclusions of this article is included within the article.

\section{Authors' contributions}

Conception/Design: LD, X-QQ, SZ, H-LZ. Collection and/or assembly of data: LD, X-QQ, H-LZ, SZ. Data analysis and interpretation: LR, LZ, KZ, W-WY, X-FW, TM, C-TZ. Manuscript writing: LD, X-QQ, H-LZ. All authors reviewed the manuscript and contributed to revising and approving the content of the final version for submission for publication.

\section{Competing interests}

The authors declare that they have no competing interests.

\section{Consent for publication}

Not applicable.

\section{Ethics approval and consent to participate}

This study was approved by the Clinical Research Ethics Committee of Tongji Hospital, Tongji Medical College, Huazhong University of Science and Technology. All participating persons were given informed consent and written informed consent was obtained from all subjects before enrollment.

Received: 3 January 2016 Accepted: 21 May 2016

Published online: 10 June 2016

\section{References}

1. Miyasaka Y, Barnes ME, Gersh BJ, Cha SS, Bailey KR, Abhayaratna WP, et al. Secular trends in incidence of atrial fibrillation in Olmsted County, Minnesota, 1980 to 2000, and implications on the projections for future prevalence. Circulation. 2006;114(2):119-25.

2. Braunschweig F, Linde C, Adamson PB, Magalski A, Erdmann E, Kjellstrom B, et al. Continuous central haemodynamic measurements during the six-minute walk test and daily life in patients with chronic heart failure. Eur J Heart Fail. 2009;11(6):594-601.

3. Zhao F, Zhang S, Chen Y, Gu W, Ni B, Shao Y, et al. Increased expression of NF-AT3 and NF-AT4 in the atria correlates with procollagen I carboxyl terminal peptide and TGF-beta1 levels in serum of patients with atrial fibrillation. BMC Cardiovasc Disord. 2014;14:167.

4. Koizumi R, Funamoto K, Hayase T, Kanke Y, Shibata M, Shiraishi Y, et al. Numerical analysis of hemodynamic changes in the left atrium due to atrial fibrillation. J Biomech. 2015;48(3):472-8.

5. Crepaz R, Romeo C, Montanaro D, De Santis S. Long-term results of treatment with bosentan in adult Eisenmenger's syndrome patients with Down's syndrome related to congenital heart disease. BMC Cardiovasc Disord. 2013;13:74

6. Stoller O, de Bruin ED, Knols RH, Hunt KJ. Effects of cardiovascular exercise early after stroke: systematic review and meta-analysis. BMC Neurol. 2012;12:45.

7. Moshkovitz Y, Kaluski E, Milo O, Vered Z, Cotter G. Recent developments in cardiac output determination by bioimpedance: comparison with invasive cardiac output and potential cardiovascular applications. Curr Opin Cardiol. 2004;19(3):229-37.

8. Tonelli AR, Alnuaimat H, Li N, Carrie R, Mubarak KK. Value of impedance cardiography in patients studied for pulmonary hypertension. Lung. 2011;189(5):369-75.

9. Sodolski T, Kutarski A. Impedance cardiography: A valuable method of evaluating haemodynamic parameters. Cardiol J. 2007;14(2):115-26.

10. Gilewski W, Banach J, Fares I, Walukiewicz M, Karasek D, Sinkiewicz W. Use of transthoracic impedance cardiography and tissue Doppler echocardiography in the cardiovascular assessment of atrial fibrillation patients subjected to electroversion. Kardiol Pol. 2013;71(4):373-80.

11. January CT, Wann LS, Alpert JS, Calkins H, Cigarroa JE, Cleveland JC, et al. 2014 AHA/ACC/HRS guideline for the management of patients with atrial fibrillation: executive summary: a report of the American College of Cardiology/American Heart Association Task Force on practice guidelines and the Heart Rhythm Society. Circulation. 2014;130(23):2071-104.

12. ATS Committee on Proficiency Standards for Clinical Pulmonary Function Laboratories. ATS statement: guidelines for the six-minute walk test. Am J Respir Crit Care Med. 2002;166(1):111-7.

13. Enright $\mathrm{PL}$, Sherrill DL. Reference equations for the six-minute walk in healthy adults. Am J Respir Crit Care Med. 1998;158(5 Pt 1):1384-7. 
14. Fortin J, Habenbacher W, Heller A, Hacker A, Grullenberger R, Innerhofer J, et al. Non-invasive beat-to-beat cardiac output monitoring by an improved method of transthoracic bioimpedance measurement. Comput Biol Med. 2006;36(11):1185-203.

15. Pu J, Mintz GS, Biro S, Lee JB, Sum ST, Madden SP, et al. Insights into echo-attenuated plaques, echolucent plaques, and plaques with spotty calcification: novel findings from comparisons among intravascular ultrasound, near-infrared spectroscopy, and pathological histology in 2,294 human coronary artery segments. J Am Coll Cardiol. 2014;63(21):2220-33.

16. Pu J, Mintz GS, Brilakis ES, Banerjee S, Abdel-Karim AR, Maini B, Biro S, et al. In vivo characterization of coronary plaques: novel findings from comparing greyscale and virtual histology intravascular ultrasound and near-infrared spectroscopy. Eur Heart J. 2012;33(3):372-83.

17. Wu XF, Yang M, Qu AJ, Mintz GS, Yang Y, Shang YP, et al. Level of Pregnancy-associated Plasma Protein-A Correlates With Coronary Thin-cap Fibroatheroma Burden in Patients With Coronary Artery Disease: Novel Findings From 3-Vessel Virtual Histology Intravascular Ultrasound Assessment. Medicine. 2016;95(3):e2563.

18. Xiajuan Z, Ding D, Yanyan H, Zhen H. Impedance cardiographic hemodynamic variables and hypertension in elderly Han residents. Upsala Journal of Medical Sciences. 2013;118(2):80-6.

19. Aoka $Y$, Hagiwara N, Kasanuki H. Heterogeneity of hemodynamic parameters in untreated primary hypertension, and individualization of antihypertensive therapy based on noninvasive hemodynamic measurements. Clinical and Experimental Hypertension (New York, Ny: 1993). 2013;35(1):61-6.

20. Rubim VS, Drumond Neto C, Romeo JL, Montera MW. [Prognostic value of the Six-Minute Walk Test in heart failure]. Arq Bras Cardiol. 2006;86(2):120-5.

21. Ventura HO, Taler SJ, Strobeck JE. Hypertension as a hemodynamic disease: the role of impedance cardiography in diagnostic, prognostic, and therapeutic decision making. Am J Hypertens. 2005;18(2 Pt 2):26S-43S.

22. Cahalin LP, Arena R, Guazzi M. Comparison of heart rate recovery after the six-minute walk test to cardiopulmonary exercise testing in patients with heart failure and reduced and preserved ejection fraction. Am J Cardiol. 2012;110(3):467-8.

23. Thomas S, Rich MW. Epidemiology, pathophysiology, and prognosis of heart failure in the elderly. Heart Fail Clin. 2007;3(4):381-7.

24. Nilsson $G$, Hedberg $P$, Jonason $T$, Lonnberg I, Ohrvik J. Heart rate recovery is more strongly associated with the metabolic syndrome, waist circumference, and insulin sensitivity in women than in men among the elderly in the general population. Am Heart J. 2007;154(3):460 e461-467.

25. Rosenwinkel ET, Bloomfield DM, Arwady MA, Goldsmith RL. Exercise and autonomic function in health and cardiovascular disease. Cardiol Clin. 2001;19(3):369-87.

26. Januzzi $\mathrm{Jr} \mathrm{JL}$. The role of natriuretic peptide testing in guiding chronic heart failure management: review of available data and recommendations for use. Arch Cardiovasc Dis. 2012:105(1):40-50.

27. Havelka EG, Rzechula KH, Bryant TO, Anneken SM, Kulstad EB. Correlation between impedance cardiography and B-type natriuretic peptide levels in dyspneic patients. J Emerg Med. 2011;40(2):146-50.

28. Lyu T, Zhao Y, Zhang T, Zhou W, Yang F, et al. Natriuretic peptides as an adjunctive treatment for acute myocardial infarction: insights from the meta-analysis of 1,389 patients from 20 trials. Int Heart J. 2014;55(1):8-16.

29. Opasich C, Pinna GD, Mazza A, Febo O, Riccardi R, Riccardi PG, et al. Six-minute walking performance in patients with moderate-to-severe heart failure; is it a useful indicator in clinical practice? Eur Heart J. 2001;22(6):488-96.

30. Bittner V, Weiner DH, Yusuf S, Rogers WJ, Mclntyre KM, Bangdiwala SI, et al. Prediction of mortality and morbidity with a 6-minute walk test in patients with left ventricular dysfunction. SOLVD Investigators. JAMA. 1993;270(14):1702-7.

31. Nguyen T, Jolly U, Sidhu K, Yee R, Leong-Sit P. Atrial fibrillation management: evaluating rate vs rhythm control. Expert Rev Cardiovasc Ther. 2016;30:1-12.

32. Rienstra M, Damman K, Mulder BA, Van Gelder IC, McMurray JJ, Van Veldhuisen DJ. Beta-blockers and outcome in heart failure and atrial fibrillation: a meta-analysis. JACC Heart Fail. 2013;1(1):21-8.

33. Zeng W, Liu ZH, Li ZY, Zhang M, Cheng YJ. Digoxin Use and adverse outcomes in patients with atrial fibrillation. Medicine (Baltimore). 2016;95(12):e2949.
34. Chamaria S, Desai AM, Reddy PC, Olshansky B, Dominic P. Digoxin use to control ventricular rate in patients with atrial fibrillation and heart failure is not associated with increased mortality. Cardiol Res pract. 2015;2015(10):314041.

\section{Submit your next manuscript to BioMed Central and we will help you at every step:}

- We accept pre-submission inquiries

- Our selector tool helps you to find the most relevant journal

- We provide round the clock customer support

- Convenient online submission

- Thorough peer review

- Inclusion in PubMed and all major indexing services

- Maximum visibility for your research

Submit your manuscript at www.biomedcentral.com/submit

) Biomed Central 